\title{
Airway neutrophilia in COPD is not associated with increased neutrophil
} survival

\author{
P. Rytilä*,\#, M. Plataki*, F. Bucchieri*, M. Uddin*, G. Nong*, \\ V.L. Kinnula and R. Djukanović*
}

ABSTRACT: Neutrophilic airway inflammation is a prominent feature of chronic obstructive pulmonary disease (COPD) and correlates with disease severity. The mechanisms that determine the extent of neutrophilia could involve increased influx or prolonged survival of neutrophils. The aim of the study was to assess whether neutrophil pro-survival mechanisms are increased in the airways of subjects with COPD owing to the presence of anti-apoptotic factors in the bronchial lining fluid.

Induced sputum samples were collected from 20 subjects with stable COPD, 14 healthy smokers and 14 healthy controls. Quantification of apoptotic neutrophils was based on typical morphological cell changes. Anti-apoptotic, pro-survival activity in the sputum was studied by culturing peripheral blood neutrophils with the fluid phase of induced sputum. Apoptosis was assessed both by morphology and flow cytometry using Annexin V/7-aminoactinomycin D staining.

COPD patients and healthy smokers had significantly higher percentages of sputum neutrophils than healthy controls. However, there were no significant differences between the three subject groups in either the proportion of apoptotic neutrophils in sputum or the in vitro antiapoptotic activity detected in the sputum fluid phase.

In conclusion, prolonged survival of neutrophils in sputum is not a feature of chronic obstructive pulmonary disease and cannot explain the increased numbers of airway neutrophils in this disease.

\section{KEYWORDS: Apoptosis, chronic obstructive pulmonary disease, inflammation, neutrophils}

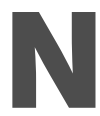

eutrophil accumulation in the airways is recognised as a prominent feature of chronic obstructive lung disease (COPD), with the extent of neutrophilic infiltration in both the airway lumen and tissues correlating with disease severity [1-4]. It has been widely hypothesised that neutrophils become activated in the airways to release reactive oxygen species, proteases, and inflammatory cytokines, which play an important role in lung injury $[5,6]$. However, little is known about the cellular and molecular mechanisms that control neutrophil migration and survival in the airways in COPD.

Neutrophil accumulation is a dynamic process that consists of the recruitment of neutrophils from the bloodstream and clearance from the lungs as a result of phagocytosis of apoptotic cells. Neutrophils are attracted from the vascular space into the airway lumen by chemotactic factors, such as interleukin (IL)-8, leukotriene $(\mathrm{LT}) \mathrm{B}_{4}$ and tumour necrosis factor- $\alpha$. Previous studies have suggested that circulating neutrophils isolated from subjects with COPD are more responsive than neutrophils from healthy control subjects to chemotactic stimuli [7]. However, it is not known whether prolonged cell survival due to reduced apoptosis of neutrophils in the airways also contributes to the accumulation of these cells.

Apoptosis is a morphologically and biochemically distinct form of cell death that limits tissue injury and is an essential requirement for the resolution of inflammation [8]. Neutrophil apoptosis has been studied extensively and shown to involve typical morphological changes, attenuation of activation and degranulation, nuclear and cytoplasmic condensation, DNA fragmentation, dilatation of the endoplasmic reticulum and alterations in cell membrane composition.
AFFILIATIONS

*Division of Infection, Inflammation and Repair, University of Southampton, Southampton, UK. "Division of Allergy, and "Division of Respiratory Diseases, Dept of Medicine, Helsinki University Central Hospital, Helsinki, Finland.

CORRESPONDENCE

P. Rytilä

Skin and Allergy Hospital Helsinki University Central Hospital Post Box 160 $00029 \mathrm{HUCH}$

Helsinki

Finland

Fax: 358947186500

E-mail: paula.rytila@hus.fi

Received:

December 192005

Accepted after revision:

August 042006

SUPPORT STATEMENT

P. Rytilä is the recipient of a European Respiratory Society fellowship (LTRF2003-003).

European Respiratory Journal Print ISSN 0903-1936 Online ISSN 1399-3003 
Further characteristic features are activation of caspases and leakage of cytochrome $c$ from the mitochondria [9, 10]. Apoptotic neutrophils are removed by macrophages using phagocyte recognition mechanisms [8]. Dysregulation of neutrophil apoptosis leads to the accumulation of neutrophils within the tissue, persistence of inflammation and increased potential for tissue damage. A variety of pro-inflammatory cytokines and other mediators have been demonstrated to delay neutrophil apoptosis in vitro; these include granulocyte macrophage-colony stimulating factor (GM-CSF), IL-8, and $\mathrm{LTB}_{4}$, all of which have been shown to be increased in the airways of subjects with COPD [11].

Little is known about the role of neutrophil apoptosis in COPD. Two studies that examined the spontaneous apoptosis of peripheral blood neutrophils $[12,13]$ found no difference in the rate of in vitro apoptosis of circulating neutrophils from subjects with stable COPD when compared with healthy smokers or healthy control subjects. The present authors have hypothesised that the presence of elevated concentrations of pro-neutrophil factors in the airways prolongs neutrophil survival, resulting in fewer apoptotic neutrophils being seen in the sputum of subjects with COPD. To test this hypothesis, induced sputum, a medium that samples the airway lining fluid and is rich in neutrophils and mediators that have a potential to delay neutrophil apoptosis [1], was used. Further evidence of altered survival of neutrophils in COPD airways was sought by studying the ex vivo anti-apoptotic effects of the sputum fluid phase on blood neutrophils.

\section{METHODS}

\section{Subjects}

A total of 20 subjects with COPD, 14 healthy smokers and 14 healthy control subjects were recruited (table 1). COPD was diagnosed according to Global Initiative for Chronic Obstructive Lung Disease (GOLD) guidelines [14]. All the COPD subjects and healthy smokers were current smokers, with mean 39 and 30 pack-yrs, respectively. Healthy smokers were free of chronic respiratory symptoms and had normal spirometry. Healthy control subjects were nonsmokers without significant past or current respiratory symptoms and with normal lung function. None of the subjects had experienced any signs of a respiratory infection in the 4 weeks preceding the study and none were treated with inhaled or oral steroids. None of the subjects had any other chronic disease.

The COPD subjects were classified into GOLD severity categories (stages $0-\mathrm{IV}$ ) on the basis of their post-bronchodilator forced expiratory volume in one second (FEV1)/forced vital capacity (FVC) and FEV1 \% predicted. COPD stage 0 subjects $(n=8)$ were current smokers with chronic cough and sputum production but normal lung function (FEV1 $>80 \%$ pred and FEV1/FVC >0.7). The other COPD subjects were classified into GOLD stage I-IV severity categories if their post-bronchodilator FEV1/FVC was $<0.7$ and their FEV1 fell into set bands (stage 1: FEV1 $\geqslant 80 \%$; stage 2 : $50 \% \leqslant$ FEV1 $<80 \%$; stage 3: $30 \% \leqslant$ FEV $1<50 \%$; stage 4 : FEV $1<30 \%$ ). Three subjects were in stage I, eight subjects were in stage II and one subject was in stage IV. Transfer factor of the lung for carbon monoxide (DL,CO) was measured by standard methods [15].

Sputum samples for both cytological analyses and in vitro experiments with cultured neutrophils were obtained from all subjects. For in vitro experiments, neutrophils were isolated from four separate individuals with no smoking history or lung disease.

The study was approved by the ethics committees of the Southampton University Hospital Trust (Southampton, UK) and Helsinki University Hospital (Helsinki, Finland). All subjects gave full informed consent.

\section{Sputum induction and processing}

Sputum induction was conducted using the guidelines of the European Respiratory Society Task Force [16]. A standard procedure of induction was conducted using $4.5 \%$ hypertonic saline given at 5-min intervals for a maximum of $20 \mathrm{~min}$, with mean induction times being similar in the three subject groups. The mucoid components of sputum were selected in order to reduce salivary contamination, and were processed as previously described [17] with slight modifications. Briefly, expectorated samples were divided into two parts: one part was treated by adding four volumes of PBS; the other was processed with four volumes of dithioerythritol (DTE). Both suspensions were filtered through $70-\mu \mathrm{m}$ nylon gauze and centrifuged at $400 \times g$ at $4{ }^{\circ} \mathrm{C}$ for $10 \mathrm{~min}$. The DTE-processed samples were used to make cytospins for total and differential cell counts. The fluid phase of the PBS-processed samples was

\section{TABLE 1 Patient characteristics}

\begin{tabular}{lccc} 
& COPD & Healthy smokers & Healthy controls \\
\hline Patients n & 20 & 14 & 14 \\
Sex male/female & $9 / 11$ & $9 / 5$ & $9 / 5$ \\
Age yrs & $55(41-72)$ & $50(22-64)$ & $44(20-62)$ \\
Smoking pack-yrs & $39(17-75)$ & $30(5-60)$ & \\
Post-bronchodilator FEV $\mathbf{1} \%$ pred & $78(25-109)^{\#}$ & $93(72-110)$ & $105(86-128)$ \\
Post-bronchodilator FEV $\mathbf{1} / \mathbf{F V}$ \% & $66(28-87)^{\star \star}$ & $78(71-88)$ & $79(70-94)$ \\
DL,CO/VA \% pred & $53(28-82)^{\star \star}$ & $75(50-96)$ & Not done \\
\hline
\end{tabular}

Data are presented as $\mathrm{n}$ for patients and sex, as median (range) for age and smoking and as mean (range) for lung function. COPD: chronic obstructive pulmonary disease; FEV1: forced expiratory volume in one second; \% pred: \% predicted; FVC: forced vital capacity; DL,CO: transfer factor of the lung for carbon monoxide; VA: alveolar volume. ${ }^{*}: \mathrm{p}<0.0001 ;{ }^{* *}: \mathrm{p}<0.01$. 
aliquoted and used for the in vitro apoptosis assays. The total protein contents of sputum supernatants were measured using the Bradford protein assay (Bio-Rad, Hercules, CA, USA) in order to adjust the in vitro results. For cell differential counts, 400 nonsquamous cells were counted on cytospins stained by the Diff-Quick method [18].

\section{Assessment of apoptotic neutrophils}

For in vitro assays only, neutrophil apoptosis was assessed by morphological criteria and flow cytometry. The morphological criteria were the disappearance of chromatin bridges between nuclear lobes (early apoptosis), and shrinkage or fragmentation of the nucleus (late apoptosis; fig. 1). The percentage of apoptotic neutrophils was assessed by counting 400 neutrophils. This was done by two independent observers with good interobserver reproducibility (intraclass correlation coefficient $0.80)$. The results were, therefore, expressed as the mean of two counts obtained by the two observers.

Apoptosis was also assessed by flow cytometry, using the technique of VERMES et al. [19], in which binding of Annexin V $(\mathrm{AxV})$ detects phosphatidylserine externalised on the outer leaflet of the cell membrane of apoptotic cells. Briefly, neutrophils were washed twice in cold PBS and were then resuspended in binding buffer (10 mM HEPES pH 7.4; $140 \mathrm{mM} \mathrm{NaCl} ; 2.5 \mathrm{mM} \mathrm{CaCl}_{2}$ ) at a density of $1 \times 10$ cells. $100 \mu \mathrm{L}^{-1}$, in $5 \mathrm{~mL}$ propylene fluorescence-activated cell sorting (FACS) tubes. AxV-phycoerythrin (PE; $1 \mu \mathrm{g} \cdot \mathrm{mL}^{-1}$ ) and 7aminoactinomycin D (7-AAD; $2.5 \mu \mathrm{g} \cdot \mathrm{mL}^{-1}$ ) were added to the tubes, which were then incubated in the dark for $15 \mathrm{~min}$. Following this, $300 \mu \mathrm{L}$ cold binding buffer was added and cells analysed using a FACScan flow cytometer (Becton and Dickinson, Oxford, UK). Control tubes lacking AxV-PE, 7AAD or both were included. Analysis of dot-plots of fluorescence channel (fl) 2 (AxV-PE) versus fl3 (7-AAD) was performed using WinMDI $2.8^{\mathrm{TM}}$ (Scripps Research Institute, La Jolla, CA, USA). The degree of early apoptosis was shown as the number of AxV-positive/7-AAD-negative cells, expressed as a percentage of total cells. AxV-negative/7-AAD-negative cells were counted as viable cells, whereas AxV-positive/
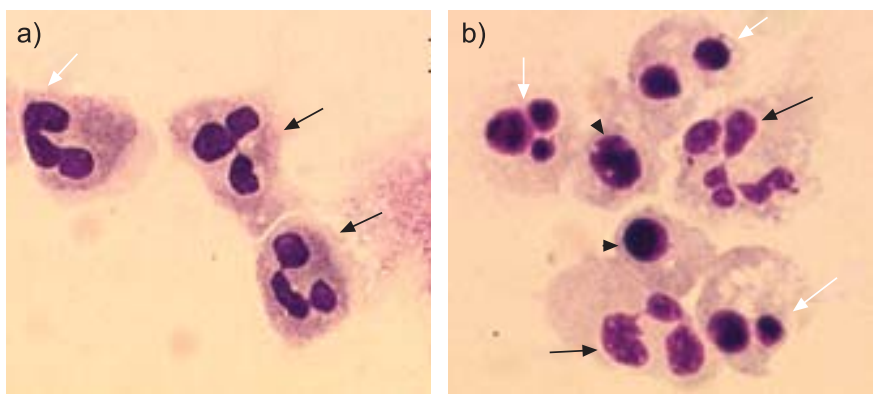

FIGURE 1. Sputum neutrophils. a) Normal neutrophils have at least two nuclear lobes interconnected by thin chromatin bridges (black arrows). Cells undergoing early apoptosis lose the chromatin bridges (white arrow). b) Neutrophils after $22 \mathrm{~h}$ in culture. Two normal neutrophils can be seen (black arrows). In cells undergoing late apoptosis (black arrowheads), there is considerable shrinkage of the cell nucleus due to condensation of nuclear chromatin. Occasionally, in late apoptotic neutrophils (white arrows), there is also fragmentation of the nucleus resulting in distinct apoptotic bodies.
7-AAD-positive and AxV-negative/7-AAD-positive cells were counted together as late apoptotic and necrotic cells.

\section{Anti-apoptotic effects of induced sputum fluid phase on cultured blood neutrophils}

The ability of sputum constituents to modulate neutrophil survival in vitro was investigated by culturing peripheral blood neutrophils purified from heparinised blood for $22 \mathrm{~h}$ (the timepoint being identified in pilot experiments as being optimal) in the presence or absence of PBS-processed sputum fluid phase. A period of $22 \mathrm{~h}$ was chosen for all experiments because the signal is consistent at this time-point, with a median reduction in survival of $68 \%$ when neutrophil cells are incubated with sputum supernatants.

GM-CSF was used as a positive control because of its known ability to reduce apoptosis in vitro [8]. PBS was used as a negative control. All the sputum fluid-phase samples were pretreated by irradiation with a dosage of $100 \mathrm{~Gy}$ to prevent bacterial growth which could affect the survival of neutrophils in culture. Heparinised venous blood was obtained from four healthy volunteers and neutrophils were isolated by dextran sedimentation/Lymphoprep (Axis-Shield, Oslo, Norway) centrifugation at $800 \times g$ for $25 \mathrm{~min}$ at $20^{\circ} \mathrm{C}$. The removal of contaminating erythrocytes was achieved by hypotonic lysis. The remaining granulocytes were washed with culture medium at $400 \times g$ for $10 \mathrm{~min}$ at $4^{\circ} \mathrm{C}$. The purity of the neutrophil population was routinely $>96 \%$. Cells were resuspended at $4 \times 10^{6}$ cells $\cdot \mathrm{mL}^{-1}$ in RPMI 1640, supplemented with $20 \%$ volume/volume (v/v) foetal calf serum (FCS; TCS Biologicals, Buckingham, UK), $4 \mathrm{mM}$ L-glutamine, $2 \mathrm{mM}$ sodium pyruvate, $100 \mathrm{U} \cdot \mathrm{mL}^{-1}$ penicillin and $100 \mu \mathrm{g} \cdot \mathrm{mL}^{-1}$ streptomycin (all from Invitrogen, Paisley, UK). Neutrophils were then incubated in Falcon tubes (Becton and Dickinson) at $37^{\circ} \mathrm{C}$ in a $5 \% \mathrm{CO}_{2}$ atmosphere (final volume $0.5 \mathrm{~mL} ; 4 \times 10^{6}$ cells). The experiments with cultured neutrophils were carried out in the presence or absence of: sputum supernatants of healthy controls, healthy smokers and patients with COPD; GM-CSF (10 ng. $\mathrm{mL}^{-1}$; R\&D Systems, Oxford, UK); and PBS as control. In preliminary studies, serial dilutions (1:10, 1:20 and 1:40) of sputum supernatants were tested for their effect on neutrophil survival in order to find the concentration which gives the maximum effect. The percentage of apoptotic cells in medium alone (in the absence of sputum supernatant) was $38.5 \%$. This was reduced to $28-30 \%$ when using the $1: 10$ dilution, $21-30 \%$ when using 1:20 dilution and 33-35\% when using the 1:40 dilution. The maximum reduction was, therefore, seen at the 1:20 dilution of sputum. Such effects were comparable to the anti-apoptotic actions of $10 \mathrm{ng} \cdot \mathrm{mL}^{-1}$ lipopolysaccharide (which reduced the spontaneous apoptosis of neutrophils to $20.6 \%$ ). This optimal dilution was, therefore, used in all subsequent experiments.

After 22-h culture, cells were harvested by centrifugation at $300 \times g$ for $10 \mathrm{~min}$ at $4{ }^{\circ} \mathrm{C}$, washed twice and resuspended in PBS containing $10 \%(\mathrm{v} / \mathrm{v})$ heat-inactivated FCS. Apoptotic neutrophils were detected by morphology from cytospins and by flow cytometry as described above. The results were expressed as a percentage of the apoptosis observed in the PBS-treated samples, to account for variability between experiments. 


\begin{tabular}{|c|c|c|c|c|}
\hline & COPD & Healthy smokers & Healthy controls & $p$-value \\
\hline Total cell count $10^{6} \cdot \mathrm{mg}^{-1}$ sputum & $2.8(0.9-17)$ & $2.2(0.4-4.4)$ & $1.8(0.3-19)$ & NS \\
\hline Viability \% & $84(60-100)$ & $91(50-100)$ & 92 (88-95) & NS \\
\hline \multicolumn{5}{|l|}{ Neutrophils } \\
\hline$\%$ & $58(36-89)$ & $54(28-79)$ & $30(0-61)$ & $<0.0001$ \\
\hline \multicolumn{5}{|l|}{ Macrophages } \\
\hline $10^{6} \cdot g^{-1}$ & $0.46(0.08-1.27)$ & $0.50(0.07-2.26)$ & $0.82(0.23-5.6)$ & NS \\
\hline$\%$ & 37 (8.3-63) & $43(20-71)$ & 63 (37-95) & 0.001 \\
\hline Eosinophils \% & $0.2(0-3.0)$ & $0(0-2.0)$ & $0(0-1.0)$ & NS \\
\hline Lymphocytes \% & $0.5(0-2.0)$ & $0(0-2.0)$ & $0.4(0-2.8)$ & NS \\
\hline
\end{tabular}

Data are presented as median (range), unless otherwise stated. Cell counts are expressed as absolute counts per weight of sputum and as percentages of total inflammatory cells, excluding squamous cells. COPD: chronic obstructive pulmonary disease; Ns: not significant.

\section{Statistics}

As the data were not normally distributed, nonparametric tests were used. Data for individual variables from the several groups were first analysed using the Kruskal-Wallis test, followed by the Mann-Whitney U-test. Correlations between variables were sought using the Spearman rank correlation test. A p-value of $<0.05$ was considered statistically significant.

\section{RESULTS}

\section{Sputum cell differential counts and sputum neutrophil apoptosis}

Adequate sputum samples were obtained from all the subjects. The median percentages of squamous cell contamination were $2.5,3.6$ and $6.2 \%$, respectively, for COPD patients, healthy smokers and healthy controls $(\mathrm{p}>0.5)$. Viabilities, as assessed by trypan blue, were 84,91 and $92 \%$, respectively, for COPD

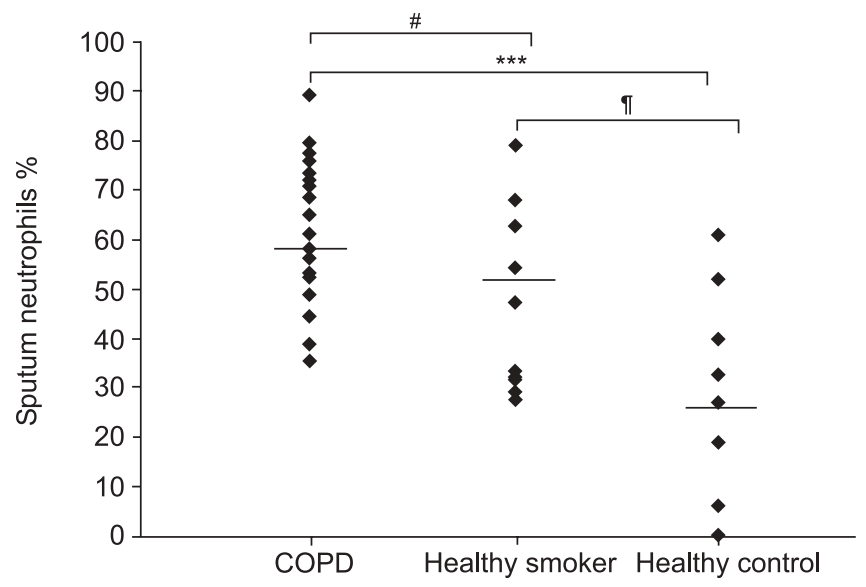

FIGURE 2. Sputum neutrophil counts (shown as percentages of nonsquamous cells). COPD: chronic obstructive pulmonary disease. ${ }^{\#}: p=0.06 ;{ }^{\circ}: p=0.01$; $\star * *: p<0.001$. patients, healthy smokers and healthy controls $(\mathrm{p}>0.5)$. Subjects with COPD and healthy smokers had a significantly higher percentage than healthy control subjects of sputum neutrophils ( $p<0.0001$ and $p=0.01$, respectively; table 2; fig. 2). COPD subjects also had a significantly lower percentage of sputum macrophages than healthy control subjects $(\mathrm{p}<0.0001)$. Compared with healthy smokers, subjects with COPD had a lower percentage of sputum macrophages $(p=0.05)$ and a tendency towards a higher percentage of sputum neutrophils $(p=0.06)$. However, there were no differences between the percentages of apoptotic neutrophils on sputum slides in the different subject groups (fig. 3). A sub-analysis was performed comparing subjects with established COPD with subjects with stage 0 COPD. No significant differences between these subgroups were seen for any of the parameters of neutrophil apoptosis measured.

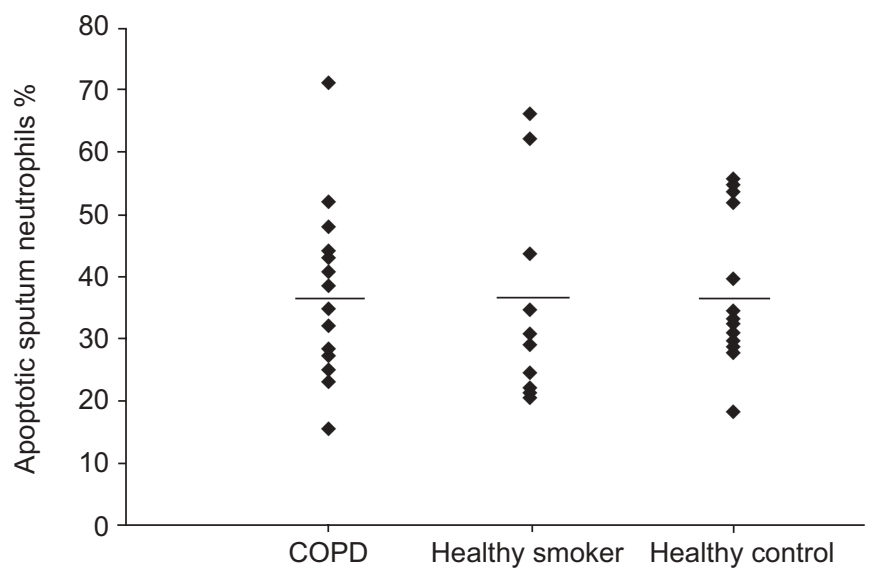

FIGURE 3. Sputum apoptotic neutrophil counts (shown as percentages of all neutrophils). Horizontal bars represent median values. COPD: chronic obstructive pulmonary disease. 

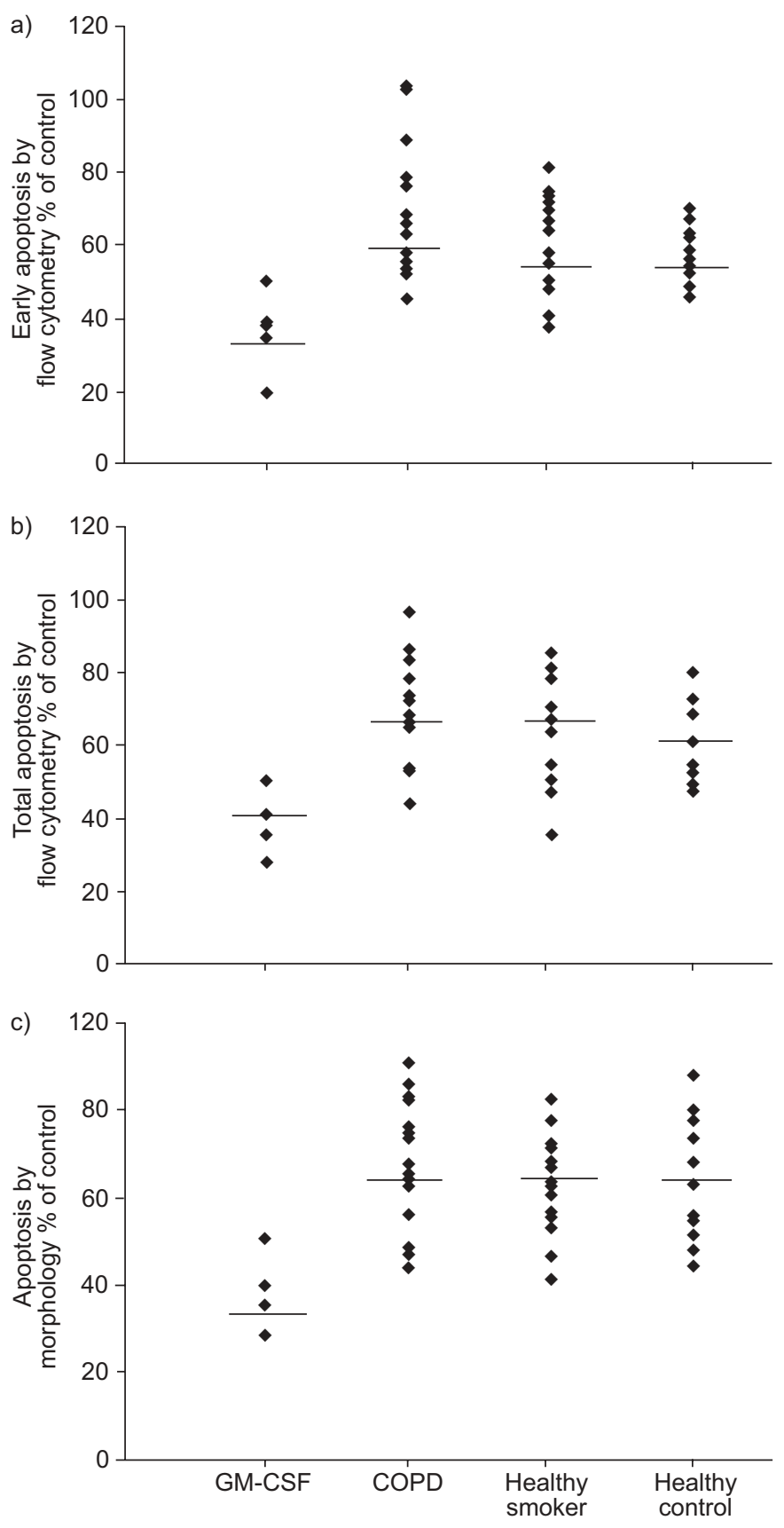

FIGURE 4. Neutrophil apoptosis after 22-h culture of blood-derived neutrophils with sputum supernatants from the three subject groups. a) Early apoptosis, measured by fluorescence-activated cell sorting (FACS); b total apoptosis, measured by FACS; and c) apoptosis, measured by morphology. Data are shown as percentages of apoptotic neutrophils seen in control culture medium and PBS alone. Horizontal bars represent median values. GM-CSF: granulocyte macrophage-colony stimulating factor; COPD: chronic obstructive pulmonary disease.

There was a significant negative correlation between pack-yrs smoked and FEV1 \% pred $\left(r_{s}=-0.47 ; p=0.005\right)$. Relative neutrophil counts correlated inversely with FEV1 \% pred $\left(\mathrm{r}_{\mathrm{s}}=-0.43 ; \mathrm{p}=0.005\right)$ and $\mathrm{DL}, \mathrm{CO} /$ alveolar volume $\left(\mathrm{r}_{\mathrm{s}}=-0.45\right.$; $\mathrm{p}=0.05)$.

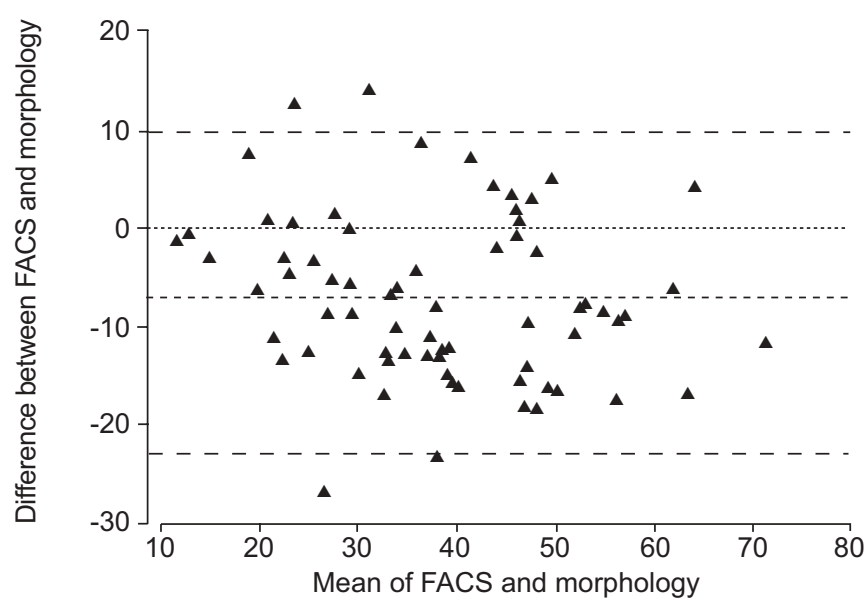

FIGURE 5. Bland-Altman plot for neutrophil apoptosis as assessed by fluorescence-activated cell sorting (FACS) and morphology. -----: mean difference between FACS and morphometry; - - -: upper and lower limits of agreement.

\section{Anti-apoptotic effects of induced sputum extract on cultured blood neutrophils}

All the sputum samples contained significant pro-survival activity for neutrophils, as demonstrated by the significant reduction in the numbers of apoptotic cells seen after $22 \mathrm{~h}$ of culture in the presence of the fluid phase of induced sputum when compared with spontaneous apoptosis in culture medium and PBS alone. However, there were no significant differences between the effects of the sputum fluid phase from nonsmoking controls, smokers and COPD patients on the extent of neutrophil apoptosis, whether assessed by low cytometry or morphology (fig. 4). There was a good correlation between the two methods (flow cytometry versus morphology) in detecting apoptosis $\left(\mathrm{r}_{\mathrm{s}}=0.70 ; \mathrm{p}<0.0001\right)$, and Bland-Altman analysis showed good agreement between the two methods (fig. 5). The positive control, GM-CSF, effectively reduced apoptosis as shown in figure 4 .

The mean total protein content of sputum was not significantly different between the three groups (COPD: $259 \mu \mathrm{g} \cdot \mathrm{mL}^{-1}$; healthy smokers: $253 \mu \mathrm{g} \cdot \mathrm{mL}^{-1}$; healthy control subjects: 181 $\left.\mu \mathrm{g} \cdot \mathrm{mL}^{-1}\right)$, and when the apoptosis data were corrected for concentrations of total protein, there were still no significant differences in the anti-apoptotic activity of sputum between the subject groups.

\section{DISCUSSION}

This study suggests that airways neutrophilia in COPD is not a result of increased pro-survival activity in the airways, i.e. of reduced pro-apoptotic mechanisms, involving soluble inflammatory mediators present in the epithelial lining fluid. While this study shows that the sputum fluid phase, which samples the bronchial lining fluid of the larger airways, invariably contains significant activity that extends the survival of neutrophils ex vivo, this activity is no different in smokers who are healthy or who have COPD, or in healthy nonsmoking subjects.

A number of studies to date have sought to elucidate the mechanisms that promote the accumulation of granulocytes in 
inflammatory airways diseases. Studies in asthma have shown that sputum contains fewer apoptotic eosinophils, the numbers of which correlate negatively with disease severity [20]. Further studies using the sputum fluid phase and either guinea pig [21] or human [22] eosinophils have attributed this effect to the cytokines GM-CSF and IL-5 [21] and prostaglandin $E_{2}$ [22]. Following the same rationale, the present authors have hypothesised that a similar mechanism could explain the airways neutrophilia that is typical of COPD. However, the present study found no significant differences in the percentages of apoptotic neutrophils in the sputum of subjects with COPD, healthy smokers and healthy control subjects. Furthermore, the rates of in vitro apoptosis of circulating neutrophils incubated with sputum supernatants obtained from all three groups were similar. Taken together, this strongly suggests that anti-apoptotic mechanisms, although operative within the airways, are not the basis of airways neutrophilia in COPD. Measuring factors such as IL-8 and GMCSF was part of the original plan, but little justification was found to explore further which chemokines/cytokines are responsible for cell survival, since no differences in cell survival were found.

Eight COPD subjects with stage 0 COPD (i.e. chronic respiratory symptoms but normal lung function) and 12 subjects with more severe COPD (eight of whom had stage II COPD) were studied. There were no significant differences in the numbers of apoptotic neutrophils or the extent of in vitro apoptosis between stage 0 COPD and more severely afflicted patients. Moreover, the numbers of apoptotic neutrophils did not correlate with lung-function parameters. Thus, it seems that the anti-apoptotic activity in the airways is not a function of COPD severity, although this cannot be ruled out for the more severe stages III and IV COPD, since only one subject with stage IV was included in the current analysis.

Apoptosis is an essential mechanism for clearing neutrophils. During apoptosis, neutrophils retain their granule contents, lose the ability to secrete them in response to secretagogues, and lose their phagocytic capacity [8]. Together with the removal of apoptotic neutrophils by macrophages, this limits tissue injury during the resolution phase of inflammation. Inefficient clearance of apoptotic cells because of either less effective or absent macrophages results in secondary necrosis and release of neutrophil activation products that are potentially toxic to the lung [8]. While the present authors have found that sputum macrophage counts, expressed as a percentage of total inflammatory cell counts, were lower in patients with COPD than in healthy control subjects, this did not seem to affect clearance since the numbers of apoptotic neutrophils were no different in COPD. Phagocytosis of dying neutrophils by macrophages before they undergo secondary necrosis and release toxic metabolites limits tissue injury, inhibits the release of pro-inflammatory cytokines and results in the production of anti-inflammatory mediators [23]. It has been shown that neutrophil elastase can cleave the phosphatidylserine receptor and impair phagocytic recognition mechanisms [24]. It remains, therefore, to be elucidated whether in COPD more neutrophils undergo secondary necrosis as opposed to apoptosis, resulting in higher concentrations of neutrophil-derived mediators such as myeloperoxidase and neutrophil elastase [22, 23].
Previous studies have shown that in vitro neutrophil apoptosis of blood neutrophils from subjects with COPD occurs at a similar rate as in healthy individuals and smokers with normal lung function [12], but is reduced during acute exacerbations of COPD and returns to levels seen in healthy control subjects within 2 weeks [13]. These observations are in keeping with studies showing reduced apoptosis of blood and bronchoalveolar lavage neutrophils in acute lung inflammation, such as respiratory distress syndrome, pneumonia $[25,26]$ and exacerbations of bronchiectasis [27]. Future studies are needed to investigate the effects of COPD exacerbations on airway neutrophil survival.

The question of why sputum neutrophil counts are increased in COPD remains unanswered. The present authors have recently shown a significant correlation between raised neutrophil counts and reduced FEV1 across the spectrum of COPD severity [4], with both the airflow limitation and sputum neutrophilia being related to the degree of small airways dysfunction, as assessed by expiratory high-resolution computed tomography lung-density measurements. A recent study by HoGG et al. [3] showed prominent inflammation in the small airways in COPD, which correlated with the decline in lung function. It is, therefore, plausible that the sputum neutrophilia that is consistently reported reflects increased accumulation of neutrophils in the small airways and their transport by the mucociliary escalator into the larger airways that are sampled by sputum induction.

In conclusion, neutrophil survival is not significantly altered in the airways of subjects with stable chronic obstructive pulmonary disease, even though their sputum contains increased numbers of neutrophils. It remains to be seen whether neutrophil influx into the airways, directed by increased chemotactic activity, is responsible for this phenomenon and to what extent chronic obstructive pulmonary disease exacerbations might affect neutrophil survival.

\section{REFERENCES}

1 Keatings VM, Collins PD, Scott DM, Barnes PJ. Differences in interleukin-8 and tumor necrosis factor-alpha in induced sputum from patients with chronic obstructive pulmonary disease or asthma. Am J Respir Crit Care Med 1996; 153: 530-534.

2 Peleman RA, Rytila PH, Kips JC, Joos GF, Pauwels RA. The cellular composition of induced sputum in chronic obstructive pulmonary disease. Eur Respir J 1999; 13: 839843.

3 Hogg JC, Chu F, Utokaparch S, et al. The nature of smallairway obstruction in chronic obstructive pulmonary disease. N Engl J Med 2004; 350: 2645-2653.

4 O'Donnell RA, Peebles C, Ward JA, et al. Relationship between peripheral airway dysfunction, airway obstruction, and neutrophilic inflammation in COPD. Thorax 2004; 59: 837-842.

5 Rahman I. Oxidative stress, chromatin remodeling and gene transcription in inflammation and chronic lung diseases. J Biochem Mol Biol 2003; 36: 95-109.

6 Repine JE, Bast A, Lankhorst I. Oxidative stress in chronic obstructive pulmonary disease. Oxidative Stress Study Group. Am J Respir Crit Care Med 1997; 156: 341-357. 
7 Burnett D, Chamba A, Hill SL, Stockley RA. Neutrophils from subjects with chronic obstructive lung disease show enhanced chemotaxis and extracellular proteolysis. Lancet 1987; 2: 1043-1046.

8 Haslett C. Granulocyte apoptosis and its role in the resolution and control of lung inflammation. Am J Respir Crit Care Med 1999; 160: S5-S11.

9 Granville DJ, Carthy CM, Hunt DW, McManus BM. Apoptosis: molecular aspects of cell death and disease. Lab Invest 1998; 78: 893-913.

10 Green DR. Introduction: apoptosis in the development and function of the immune system. Semin Immunol 2003; 15: 121-123.

11 Barnes PJ, Shapiro SD, Pauwels RA. Chronic obstructive pulmonary disease: molecular and cellular mechanisms. Eur Respir J 2003; 22: 672-688.

12 Noguera A, Sala E, Pons AR, Iglesias J, MacNee W, Agusti AG. Expression of adhesion molecules during apoptosis of circulating neutrophils in COPD. Chest 2004; 125: 1837-1842.

13 Pletz MWR, Ioanas M, de Roux A, Burkhardt O, Lode H. Reduced spontaneous apoptosis in peripheral blood neutrophils during exacerbation of COPD. Eur Respir J 2004; 23: 532-537.

14 Pauwels RA, Buist AS, Calverley PM, Jenkins CR, Hurd SS. Global strategy for the diagnosis, management, and prevention of chronic obstructive pulmonary disease. NHLBI/WHO Global Initiative for Chronic Obstructive Lung Disease (GOLD) Workshop summary. Am J Respir Crit Care Med 2001; 163: 1256-1276.

15 American Thoracic Society, Single breath carbon monoxide diffusing capacity (transfer factor). Recommendations for a standard technique. Statement of the American Thoracic Society. Am Rev Respir Dis 1987; 136: 1299-1307.

16 Djukanovic R, Sterk PJ, Fahy JV, Hargreave FE. Standardised methodology of sputum induction and processing. Eur Respir J 2002; 20: Suppl. 37, 1s-2s.

17 Hadjicharalambous C, Dent G, May RD, et al. Measurement of eotaxin (CCL11) in induced sputum supernatants: validation and detection in asthma. J Allergy Clin Immunol 2004; 113: 657-662.
18 Efthimiadis A, Spanevello A, Hamid Q, et al. Methods of sputum processing for cell counts, immunocytochemistry and in situ hybridisation. Eur Respir J 2002; 20: Suppl. 37, 19s-23s.

19 Vermes I, Haanen C, Steffens-Nakken H, Reutelingsperger C. A novel assay for apoptosis. Flow cytometric detection of phosphatidylserine expression on early apoptotic cells using fluorescein labelled Annexin V. J Immunol Methods 1995; 184: 39-51.

20 Duncan CJA, Lawrie A, Blaylock MG, Douglas JG, Walsh GM. Reduced eosinophil apoptosis in induced sputum correlates with asthma severity. Eur Respir J 2003; 22: 484-490.

21 Adachi T, Motojima S, Hirata A, Fukuda T, Makino S. Eosinophil viability-enhancing activity in sputum from patients with bronchial asthma, contributions of interleukin-5 and granulocyte/macrophage colonystimulating factor. Am J Respir Crit Care Med 1995; 151: 618-623.

22 Profita M, Sala A, Bonanno A, et al. Increased prostaglandin $E_{2}$ concentrations and cyclooxygenase-2 expression in asthmatic subjects with sputum eosinophilia. J Allergy Clin Immunol 2003; 112: 709-716.

23 Fadok VA, Bratton DL, Guthrie L, Henson PM. Differential effects of apoptotic versus lysed cells on macrophage production of cytokines: role of proteases. J Immunol 2001; 166: 6847-6854.

24 Vandivier RW, Fadok VA, Hoffmann PR, et al. Elastasemediated phosphatidylserine receptor cleavage impairs apoptotic cell clearance in cystic fibrosis and bronchiectasis. J Clin Invest 2002; 109: 661-670.

25 Droemann D, Aries SP, Hansen F, et al. Decreased apoptosis and increased activation of alveolar neutrophils in bacterial pneumonia. Chest 2000; 117: 1679-1684.

26 Matute-Bello G, Liles WC, Radella F, et al. Neutrophil apoptosis in the acute respiratory distress syndrome. Am J Respir Crit Care Med 1997; 156: 1969-1977.

27 Watt AP, Brown V, Courtney J, et al. Neutrophil apoptosis, proinflammatory mediators and cell counts in bronchiectasis. Thorax 2004; 59: 231-236. 